\title{
ABORDAGEM FISIOTERAPÊUTICA EM QUEIMADOS: REVISÃO SISTEMÁTICA
}

\author{
Tereza Cristina dos Reis FERREIRA ${ }^{1}$ \\ Layse Caroline Felix Silva e SILVA ${ }^{2}$ \\ Maria Isabel Galletti dos SANTOS ${ }^{2}$
}

1. Fisioterapeuta, Mestre em Saúde, Sociedade e Endemias na Amazônia, pela Universidade Federal do Amazonas-UFAM, Brasil. Docente do curso de fisioterapia pelo Centro Universitário do ParáCESUPA. E-mail: terezareisorientacao@yahoo.com.br;

2. Discentes em Fisioterapia pelo Centro Universitário do Pará - CESUPA. E-mail: laysenet@@ hotmail.com; misabelgalletti@hotmail.com.

\section{Recebido em: 30/05/2014 - Aprovado em: 15/09/2014 - Disponibilizado em: 15/12/2014}

\begin{abstract}
RESUMO
A queimadura pode ser definida como um trauma capaz de ocasionar variadas lesões que, de acordo com o nível, pode levar o enfermo a óbito. O fisioterapeuta atua em cada estágio da queimadura, auxiliando a cicatrização e evitando complicações. Isto vai contribuir aos índices de recuperação, reduzindo sequelas e melhorando fisicamente e psicologicamente a qualidade de vida, reintegrando o indivíduo ao convívio social. Esse trabalho teve como objetivo realizar uma revisão sistemática com intuito de oferecer a melhor informação disponível para a tomada de decisão nesse campo e identificar o maior número possível de estudos relacionados a respeito da abordagem fisioterapêutica em queimados e assim contribuir a cerca da literatura. Foi realizada uma revisão sistemática, retrospectiva e descritiva da literatura, disponível nas bases de dados LILACS e SCIELO, em língua brasileira. Foram evidenciados 15 artigos na literatura dentro dos critérios de inclusão e exclusão. Pode-se concluir que a fisioterapia atua em todas as consequências da queimadura, não apenas na parte motora e respiratória, mas também na parte psicológica dos indivíduos, tendo excelentes resultados para a recuperação do paciente queimado.
\end{abstract}

Palavras-chave: Fisioterapia. Queimaduras. Unidade de queimados. Terapia respiratória. Queimados.

\section{PHYSIOTHERAPEUTIC APPROACH IN BURNED: A SYSTEMATIC REVIEW}

\begin{abstract}
The burn can be defined as a trauma capable to cause injuries that, depending the level, can take the patient to death. The physiotherapist acts in each phase at the burn, helping the healing and avoiding complications. This is going to contribute the indices of recovering, decreasing the damages and improving the quality of life physically and psychologically, reintegrating the patient to the social life. Realize an systematic review with the intention to offer a better available information to making-decision in this field and identify the bigger number possible of related studies in respect to the physiotherapy approach in burned people and then contribute with the literature. Systematic review, retrospective and descriptive of the literature, available in the database LILACS and SCIELO, in Brazilian language. Were evidenced 15 articles in the literature with the standards for inclusion or exclusion. The physical therapy acts at all burn's consequences not just at the motor and respiratory part, but also at the psychological part of this group, having an excellent result for recovering in burn patients.
\end{abstract}

Key-words: Physiotherapy. Burns. Burn unit. Respiratory therapy. Burned. 


\section{INTRODUÇÃO}

A queimadura pode ser definida como trauma capaz de ocasionar variadas lesões que, de acordo com o nível, pode levar o enfermo a óbito, tais como, hiperemia restrita à área queimada, alterações celulares e imunológicas decorrentes do insulto, envolvimento das vias respiratórias e ocorrência de traumatismos associados (BORGES, 2010). As causas mais frequentes de queimaduras são: a chama de fogo, o contato com água fervente ou outros líquidos quentes e o contato com objetos aquecidos. Menos comuns são as queimaduras provocadas pela corrente elétrica, transformada em calor ao contato com o corpo (VALE, 2005).

O fisioterapeuta atua em cada estágio da queimadura, obtendo diversos recursos para ajudar à lesão cicatrizar de maneira correta e evitando complicações. Isto vai contribuir à recuperação, reduzindo sequelas e melhorando fisicamente e psicologicamente a qualidade de vida, propiciando ao indivíduo um melhor convívio social (SOUZA; SANTOS; OLIVATO, 2009).

Portanto o presente estudo tem como objetivo realizar uma revisão sistemática com intuito de oferecer a melhor informação disponível para a tomada de decisão nesse campo, através de um processo rigoroso e bem definido e identificar o maior número possível de estudos relacionados a respeito da abordagem fisioterapêutica em queimados e assim contribuir a cerca da literatura.

\section{METODOLOGIA}

A pesquisa caracteriza-se como revisão sistemática e descritiva. Quanto ao tipo de publicação, foram incluídos apenas os periódicos nas bases de dados Literatura Latino-Americana e do Caribe em Ciências da Saúde (LILACS) e Scientific Electronic Library Online (SciELO) e artigos publicados no período de 2000 a 2014 no idioma Português (Brasil). Os descritores utilizados foram: Unidade de queimados $x$ Fisioterapia, Queimaduras $x$ Fisioterapia, Fisioterapia, Terapia respiratória $\mathrm{X}$ Queimados $\mathrm{X}$ Fisioterapia, Serviço hospitalar de fisioterapia $\mathrm{x}$ Queimados, Modalidades de fisioterapia $\mathrm{x}$ Queimados. Foram adotados com critério de exclusão: artigos em outros idiomas que não o português e artigos publicados antes do ano de 2000. Após a leitura e fichamento dos dados, estes foram tabulados para análise e elaboração dos resultados e discussão. O período de coleta de dados foi de março a maio de 2014.

\section{RESULTADOS}

Foram evidenciados na literatura 12 artigos de acordo com os critérios de inclusão e exclusão. 
Tabela I - Artigos sobre a abordagem fisioterapêutica em queimados.

\begin{tabular}{|c|c|c|c|c|}
\hline Autores/Ano & Tipo de estudo & Amostra & Resultados & Conclusão \\
\hline $\begin{array}{l}\text { Souza, TR; } \\
\text { Santos, RT; } \\
\text { Olivatto, } \\
\text { RM } \\
2009\end{array}$ & Relato de caso & $\begin{array}{l}\text { Estudo de } \\
\text { caso }\end{array}$ & $\begin{array}{l}\text { Treinamento muscular por meio } \\
\text { de protocolo composto por } \\
\text { aumento progressivo do tempo de } \\
\text { respiração espontânea, alternado } \\
\text { com o suporte ventilatório. Após } \\
36 \text { dias de internação paciente } \\
\text { evoluiu com alta hospitalar. }\end{array}$ & $\begin{array}{l}\text { Obteve-se melhora da força muscular } \\
\text { respiratória, possibilitando retorno da } \\
\text { paciente à respiração espontânea mais } \\
\text { precocemente, diminuindo assim o } \\
\text { risco de maiores complicações } \\
\text { respiratórias associada à ventilação } \\
\text { mecânica. }\end{array}$ \\
\hline $\begin{array}{l}\text { Albuquerque } \\
\text {, MLL; } \\
\text { Silva, GPF; } \\
\text { Diniz, } \\
\text { DMSM; } \\
\text { Figueiredo, } \\
\text { AMF; } \\
\text { Câmara, } \\
\text { TMF;Bastos, } \\
\text { VPD } \\
\text { 2010 }\end{array}$ & $\begin{array}{l}\text { Estudo do tipo } \\
\text { exploratório, } \\
\text { descritivo e } \\
\text { transversal, } \\
\text { com } \\
\text { abordagem } \\
\text { quantitativa }\end{array}$ & $\begin{array}{l}20 \\
\text { pacientes }\end{array}$ & $\begin{array}{l}\text { A fisioterapia foi de grande } \\
\text { relevância para a reabilitação dos } \\
\text { pacientes, mostrando que } 85 \% \\
\text { ficaram satisfeitos com os } \\
\text { exercícios e obtiveram uma } \\
\text { melhora significativa. }\end{array}$ & $\begin{array}{l}\text { O conhecimento inerente às sequelas } \\
\text { de queimaduras é importante para a } \\
\text { identificação do impacto das } \\
\text { queimaduras em nosso meio. A } \\
\text { fisioterapia age com eficácia no } \\
\text { tratamento de pacientes queimados, } \\
\text { evitando complicações e diminuindo } \\
\text { as sequelas funcionais e estéticas. }\end{array}$ \\
\hline $\begin{array}{l}\text { Andrade, } \\
\text { AG; } \\
\text { Lima, } \\
\text { CF;Albuquer } \\
\text { que, AKB } \\
2010\end{array}$ & $\begin{array}{l}\text { Revisão } \\
\text { bibliográfica }\end{array}$ & $\begin{array}{l}\text { Levantame } \\
\text { nto } \\
\text { bibliográfi } \\
\text { co }\end{array}$ & $\begin{array}{l}\text { Essa revisão evidencia que o laser } \\
\text { terapêutico acelera a proliferação } \\
\text { das células reparativas e aumenta } \\
\text { a organização do colágeno. }\end{array}$ & $\begin{array}{l}\text { O laser terapêutico pode ser um } \\
\text { recurso valioso no tratamento de } \\
\text { queimados pela sua capacidade de } \\
\text { induzir cicatrização rápida e } \\
\text { organizada. }\end{array}$ \\
\hline $\begin{array}{l}\text { Júnior, GFP; } \\
\text { Vieira, ACP; } \\
\text { Alves, GMG } \\
2010\end{array}$ & $\begin{array}{l}\text { Descritivo e } \\
\text { transversal }\end{array}$ & $\begin{array}{l}21 \\
\text { indivíduos }\end{array}$ & $\begin{array}{l}\text { Dezesseis }(76,2 \%) \text { eram do sexo } \\
\text { masculino e cinco }(23,8) \text { do sexo } \\
\text { feminino. Todos apresentavam } \\
\text { sequelas, sendo que } 13(61,9 \%) \\
\text { sujeitos tinham sequelas tanto } \\
\text { estéticas quanto funcionais. }\end{array}$ & $\begin{array}{l}\text { As limitações físicas e psíquicas } \\
\text { causadas pela queimadura diminuem } \\
\text { a QV de quem sofre esse trauma. } \\
\text { Faz-se, portanto, cada vez mais } \\
\text { necessária a pronta e abrangente } \\
\text { reabilitação desses pacientes, para } \\
\text { minimizar os danos causados pelo } \\
\text { trauma e melhorar sua QV. }\end{array}$ \\
\hline $\begin{array}{l}\text { Silva, KP; } \\
\text { Caparróz, } \\
\text { MR; } \\
\text { Torquato, JÁ } \\
2010\end{array}$ & $\begin{array}{l}\text { Estudo } \\
\text { retrospectivo, } \\
\text { descritivo e } \\
\text { Transversal }\end{array}$ & $\begin{array}{l}\text { Análise de } \\
155 \\
\text { prontuários }\end{array}$ & $\begin{array}{l}\text { As complicações respiratórias } \\
\text { foram encontradas em 23,9\%, } \\
\text { onde a mais prevalente foi lesão } \\
\text { inalatória }(32,5 \%) \text {, sendo que } \\
80 \% \text { utilizaram ventilação } \\
\text { mecânica invasiva e } 20 \% \\
\text { ventilação mecânica não-invasiva } \\
\text { e todos fizeram uso de } \\
\text { oxigenoterapia. }\end{array}$ & $\begin{array}{l}\text { A prevalência de complicações } \\
\text { respiratórias nesta população foi por } \\
\text { lesão inalatória, seguida por } \\
\text { pneumonias. O oxigênio é muito } \\
\text { utilizado para tratamento destas } \\
\text { complicações, associado à intubação } \\
\text { orotraqueal, ventilação mecânica não } \\
\text { invasiva e nebulização. }\end{array}$ \\
\hline $\begin{array}{l}\text { Spinelli, J; } \\
\text { Rezegue, L; } \\
\text { Fiorin, R; } \\
\text { Bragança, } \\
\text { KR } \\
2010\end{array}$ & Relato de caso & $\begin{array}{l}\text { Estudo de } \\
\text { caso }\end{array}$ & $\begin{array}{l}\text { Submetido a } \\
\text { orotraqueal precoce e ventilação } \\
\text { mecânica, com controle rigoroso } \\
\text { dos sinais vitais. O paciente foi } \\
\text { extubado sem intercorrências e } \\
\text { mantido em oxigenoterapia, com } \\
\text { posterior alta para enfermaria, } \\
\text { com suporte de fisioterapia } \\
\text { respiratória. Houve retirada }\end{array}$ & $\begin{array}{l}\text { A conduta tomada de forma precoce e } \\
\text { a condução correta do tratamento da } \\
\text { lesão, tanto em sua repercussão } \\
\text { respiratória, quanto em sua } \\
\text { repercussão sistêmica, foi de grande } \\
\text { importância no que diz respeito ao } \\
\text { tratamento e sobrevida desse } \\
\text { paciente, visto que esse tipo de lesão } \\
\text { apresenta alto índice de mortalidade e }\end{array}$ \\
\hline
\end{tabular}




\begin{tabular}{|c|c|c|c|c|}
\hline & & & $\begin{array}{l}\text { gradual do suporte de oxigênio. } \\
\text { Alta hospitalar após } 23 \text { dias. }\end{array}$ & complexidade de seu tratamento. \\
\hline $\begin{array}{l}\text { Daibem, } \\
\text { CGL; } \\
\text { Conti, TGT; } \\
\text { Silva, } \\
\text { MMA; } \\
\text { Rocha, C } \\
2011\end{array}$ & $\begin{array}{l}\text { Estudo } \\
\text { descritivo e } \\
\text { prospectivo }\end{array}$ & 5 pacientes & $\begin{array}{l}\text { A média das pressões intracuff } \\
\text { encontradas nas primeiras } 24 \\
\text { horas foi } 40,53 \pm 11,8 \mathrm{cmH} 2 \mathrm{O} \text {; } \\
\text { nas } 48 \text { horas, } 21,86 \pm 3,92 \\
\text { cmH2O, e nas } 72 \text { horas, } 19,86 \pm \\
1,38 \mathrm{cmH} 2 \mathrm{O} \text {. }\end{array}$ & $\begin{array}{l}\text { As pressões alteram } \\
\text { significativamente nas primeiras } 72 \mathrm{~h} \\
\text { e que consequentemente acabam por } \\
\text { expor o paciente a riscos causados } \\
\text { por pressões inadequadas, sugerindo } \\
\text { que há relação entre o aumento da } \\
\text { pressão do cuff ocasionado pelo } \\
\text { edema e o produto do cálculo de } \\
\text { Parkland. }\end{array}$ \\
\hline $\begin{array}{l}\text { Ferreira, } \\
\text { TCR; } \\
\text { Carepa, SS; } \\
\text { Spinelli, JL; } \\
\text { Bastos, JO; } \\
\text { Costa, LR } \\
2011\end{array}$ & $\begin{array}{l}\text { Estudo } \\
\text { individualizad } \\
\text { o, } \\
\text { quantitativo, } \\
\text { observacional } \\
\text { e do tipo } \\
\text { transversal }\end{array}$ & $\begin{array}{l}11 \\
\text { pacientes }\end{array}$ & $\begin{array}{l}81,81 \% \text { pacientes eram do gênero } \\
\text { masculino e } 18,18 \% \text {, do feminino. } \\
\text { Nesse estudo foi encontrado } \\
\text { ausência de dor, discreto aumento } \\
\text { de frequência respiratória e } \\
\text { redução dos valores de PIMAX e } \\
\text { PEMAX. }\end{array}$ & $\begin{array}{l}\text { Na presente pesquisa, as alterações da } \\
\text { mecânica respiratória são } \\
\text { consideráveis, com exceção do } \\
\text { volume corrente, o qual apresentou } \\
\text { grande variação de seus valores. }\end{array}$ \\
\hline $\begin{array}{l}\text { Takejima, } \\
\text { ML; Netto, } \\
\text { RFB; } \\
\text { Toebe, BL; } \\
\text { Andretta, } \\
\text { MA; } \\
\text { Prestes, MA; } \\
\text { Takaki, JL } \\
2011\end{array}$ & $\begin{array}{l}\text { Estudo } \\
\text { prospectivo } \\
\text { realizado por } \\
\text { meio de } \\
\text { entrevista, } \\
\text { com } \\
\text { questionário }\end{array}$ & $\begin{array}{l}776 \\
\text { entrevistad } \\
\text { os }\end{array}$ & $\begin{array}{l}16,36 \% \text { dos } \\
\text { entrevistados } \\
\text { afirmaram possuir algum tipo de } \\
\text { material de prevenção domiciliar } \\
\text { contra queimaduras. } 26,67 \% \\
\text { demonstraram saber o que fazer } \\
\text { no caso de uma queimadura, } \\
31,44 \% \text { receberam alguma } \\
\text { informação decorrente de } \\
\text { campanhas públicas e } 2,44 \% \\
\text { obtiveram algum dado sobre } \\
\text { queimadura enquanto aguardava } \\
\text { atendimento na Unidade de } \\
\text { Saúde. }\end{array}$ & $\begin{array}{l}\text { Apesar da eficácia das ações } \\
\text { preventivas ser conhecida, os } \\
\text { pacientes não recebem informações } \\
\text { sobre queimaduras nem mesmo nas } \\
\text { unidades de saúde, como os Centros } \\
\text { Municipais de Urgências Médicas de } \\
\text { Curitiba. }\end{array}$ \\
\hline $\begin{array}{l}\text { Lyra, MC; } \\
\text { Júnior, HL; } \\
\text { Neto, SP; } \\
\text { Orgaes, } \\
\text { FAFS; } \\
\text { Gonella, HÁ } \\
2012\end{array}$ & $\begin{array}{l}\text { Protocolo de } \\
\text { manuseio de } \\
\text { animais, } \\
\text { seguindo as } \\
\text { normas pré- } \\
\text { estabelecidas } \\
\text { pelo Colégio } \\
\text { Brasileiro de } \\
\text { Experimentaçã } \\
\text { o Animal } \\
\text { (COBEA). }\end{array}$ & $24 \mathrm{r}$ & $\begin{array}{l}\text { Os ratos foram queimados em } \\
\text { região dorsal por imersão em } \\
\text { água a } 70^{\circ} \mathrm{C} \text {, queimaduras de } \\
\text { segundo grau e divididos em } 3 \\
\text { grupos: - Grupo A - grupo } \\
\text { controle; } \\
\text { - Grupo B - duas sessões por } \\
\text { semana de caboxiterapia até } 4 \\
\text { semanas; } \\
\text { - Grupo C - três sessões por } \\
\text { semana de carboxiterapia até } 4 \\
\text { semanas. }\end{array}$ & $\begin{array}{l}\text { O presente trabalho não apresenta } \\
\text { diferenças estatísticas entre a } \\
\text { carboxiterapia e o grupo controle. }\end{array}$ \\
\hline $\begin{array}{l}\text { Santana, } \\
\text { CML; } \\
\text { Brito, CF; } \\
\text { Costa, } \\
\text { ACSM } \\
2012\end{array}$ & $\begin{array}{l}\text { Estudo de } \\
\text { intervenção e } \\
\text { de campo, } \\
\text { com a } \\
\text { natureza } \\
\text { qualitativa } \\
\text { e quantitativa }\end{array}$ & $\begin{array}{l}30 \\
\text { voluntários }\end{array}$ & $\begin{array}{l}\text { Antes da fisioterapia, a fase } \\
\text { predominante foi a inflamatória e, } \\
\text { após, foi a de remodelação. O } \\
\text { edema regrediu em todos os } \\
\text { pacientes após a terapia. Houve } \\
\text { aumento significativo em todas as } \\
\text { variáveis estudadas. }\end{array}$ & $\begin{array}{l}\text { Os parâmetros clínicos } \\
\text { comparados antes e após a submissão } \\
\text { dos pacientes ao tratamento } \\
\text { fisioterapeutico apresentaram valor } \\
\text { preditivo significativo em todas as } \\
\text { variáveis, ratificando importância } \\
\text { desse serviçona reabilitação. }\end{array}$ \\
\hline $\begin{array}{l}\text { Guimarães, } \\
\text { IBA; } \\
\text { Martins, }\end{array}$ & $\begin{array}{l}\text { Estudo } \\
\text { exploratório, } \\
\text { de abordagem }\end{array}$ & $\begin{array}{l}20 \\
\text { pacientes }\end{array}$ & $\begin{array}{l}\text { Na avaliação da QV, os melhores } \\
\text { resultados foram encontrados nos } \\
\text { domínios "Vitalidade" }\end{array}$ & $\begin{array}{l}\text { O significado positivo de qualidade } \\
\text { de vida relaciona-se, prioritariamente, } \\
\text { aos domínios "Vitalidade" e ao }\end{array}$ \\
\hline
\end{tabular}




\begin{tabular}{|c|c|c|c|}
\hline $\begin{array}{l}\text { ABT; } \\
\text { Guimarães, } \\
\text { SB } \\
2013\end{array}$ & $\begin{array}{l}\text { analítico- } \\
\text { descritiva e } \\
\text { corte } \\
\text { transversal }\end{array}$ & $\begin{array}{l}\text { "Saúde mental" (57,6), enquanto } \\
\text { que os piores resultados foram } \\
\text { identificados nos domínios } \\
\text { "Aspectos físicos" (0) e "Aspectos } \\
\text { emocionais" (0). }\end{array}$ & $\begin{array}{l}\text { "Estado mental" para os pacientes } \\
\text { internados na CTQ do IJF. Os } \\
\text { domínios com } \\
\text { comprometimento são "Aspectos } \\
\text { físicos" e "Aspectos emocionais", } \\
\text { refletindo a percepção de sua } \\
\text { autoimagem diante das queimaduras. }\end{array}$ \\
\hline
\end{tabular}

Fonte: Dos autores.

\section{DISCUSSÃO}

$\mathrm{O}$ agente causal e a circunstância da queimadura produzem em cada indivíduo efeitos diferentes, então a equipe multiprofissional deve utilizar uma linguagem comum e funcionar como um suporte, envolvendo o paciente no seu processo de reabilitação. A abordagem fisioterapêutica no queimado tem dois fundamentos: a avaliação do potencial de sequela da queimadura e avaliação da condição psicossocial do indivíduo. Esses dois são importantes para que se estabeleça uma relação de colaboração e envolvimento do paciente em sua reabilitação. A nível hospitalar a atuação do fisioterapeuta deve ser precoce e contínua, desde a fase de internação a alta dada pelo cirurgião plástico, coincidindo com a alta fisioterapêutica (BORGES, 2010).

De acordo com os levantamentos realizados, a principal complicação respiratória citada pelos autores foi a lesão inalatória, sendo o oxigênio muito utilizado para o tratamento dessas complicações. Souza et al (2004), relatam que a lesão inalatória é considerada a principal causa de morte nos pacientes queimados e em todos os casos de suspeita de intoxicação, está indicado o uso de altas frações de oxigênio. Os mecanismos envolvidos na gênese da lesão inalatória envolvem tanto os fatores de ação local quanto os de ação sistêmica, o que acaba por aumentar muito as repercussões da lesão.

Os resultados deste estudo mostraram que para pacientes com lesão inalatória, a conduta tomada de forma precoce e a condução correta do tratamento da lesão, tanto na repercussão respiratória quanto sistêmica, foi de grande importância no que diz respeito ao tratamento e sobrevida do paciente. Este tipo de lesão apresenta alto índice de mortalidade e complexidade de seu tratamento. A ênfase no tratamento precoce mesmo em pacientes sem quadro clínico de insuficiência respiratória teve impacto na evolução do paciente com lesão inalatória. Souza et al (2004) relataram que o ponto central na terapêutica dos pacientes vítimas de grandes queimaduras com lesões inalatórias é a compreensão da grande resposta inflamatória existente, com suas repercussões 
pulmonares e sistêmicas como um fenômeno global e não como complicações isoladas.

Nos resultados, em pacientes com curativos oclusivos em tórax pode-se perceber que houve alterações respiratórias significativas. Houve aumento da frequência respiratória pela compensação para manter um volume minuto adequado. Houve também alterações significativas dos valores da pressão inspiratória máxima (PIMAX) e pressão expiratória máxima (PEMAX), devido a diminuição de força muscular causada por diminuição de massa e inatividade, feito que corrobora com o estudo de Torquato et al (2009), onde o curativo oclusivo levou à diminuição da força muscular inspiratória e expiratória, queda no fluxo expiratório e na capacidade vital. $\mathrm{O}$ volume minuto e a frequência respiratória aumentaram, porém não de maneira significativa. O curativo oclusivo pode intensificar ainda mais a restrição da caixa torácica imposta pela própria afecção e diminuir a força muscular e os volumes pulmonares, contribuindo para maiores áreas de atelectasias e maior probabilidade da ocorrência de complicações pulmonares.

Os resultados encontrados mostram que as pressões de cuff nas primeiras 72 horas alteram significativamente e acabam por expor os pacientes a riscos causados por pressões inadequadas sugerindo que há relação entre o aumento da pressão do cuff ocasionado pelo edema e o produto do cálculo de Parkland. O estudo de Juliano et al (2007), mostra que as medidas do cuff foram irregulares em $80 \%$ dos casos, sugerindo-se a necessidade de vigilância das pressões do balonete através da implantação de uma rotina de mensurações, para prevenir complicações como broncoaspiração em pressões menores e lesões na parede da traqueia em pressões maiores que o ideal.

Os resultados mostram que as limitações físicas e psíquicas causadas pela queimadura diminuem a qualidade de vida de quem sofre esse trauma. No estudo de Costa et al (2009), os participantes apresentaram grande descontentamento com a aparência física alterada pelas sequelas da queimadura, evidenciando tentativas de escondê-las. Faz-se, portanto, cada vez mais necessária, a pronta e abrangente reabilitação desses pacientes, para minimizar os danos causados pelo trauma e melhorar a qualidade de vida. A atividade física precoce para este individuo é de extrema importância para a manutenção da amplitude articular.

Nos artigos analisados o protocolo fisioterapêutico mostrou-se eficaz, pois conseguiu obter melhora da força muscular respiratória, possibilitando retorno da paciente à respiração espontânea mais precocemente, diminuindo assim o risco de maiores 
complicações respiratórias associada à ventilação mecânica; também foi possível observar nos resultados uma evolução satisfatória, por meio do ganho de ADM e força muscular, melhorando a reabilitação não só física mas também psicológica do indivíduo. Os parâmetros clínicos comparados antes e após a submissão dos pacientes ao tratamento fisioterapêutico apresenta valor significativo na maioria das variáveis, ratificando a importância desse serviço ao paciente vítima de queimadura. O estudo de Borges (2010), mostra algumas técnicas fisioterapêuticas utilizadas no paciente queimado ainda no período de internação hospitalar, que são na parte respiratória: técnicas de broncodesobstrução, desinsuflação pulmonar, reexpansão pulmonar, reeducação da função muscular respiratória e prevenção das deformidades torácicas subsequentes à lesão. Quanto a reabilitação motora, os exercícios são: exercício passivo, para redução de edema, preservação ou recuperação da integridade articular, prevenindo rigidez articular e encurtamentos de tecido moles; exercícios ativos assistidos para aumentar amplitude de movimento; exercícios ativos, estimulando o paciente a executar sozinho os movimentos, vencendo a gravidade; exercícios resistidos para recuperar força e massa muscular; exercícios isométricos, para manter massa muscular; estiramentos, para alongamento de fibras musculares. Após a sessão fisioterapêutica, deve-se observar a manutenção dos ganhos obtidos nos exercícios. O estudo de Medeiros (2010), relata que quando bem empregada, a fisioterapia traz resultados satisfatórios e contribuem muito para a recuperação do paciente.

Pelos resultados encontrados o laser terapêutico é um recurso valioso para esse tipo de paciente, pela capacidade de induzir a cicatrização rápida e organizada. Lamberti et al (2014), concorda com os efeitos e benefícios do laser terapêutico como forma de tratamento na cicatrização de um paciente queimado. $\mathrm{O}$ resultado de um estudo com ratos evidenciou que o grupo submetido à carboxiterapia e o grupo controle não tiveram diferenças estatísticas em seus achados. Porém, Borges (2010), relata que a carboxiterapia atua como coadjuvante nos pacientes com sequelas de queimaduras, proporcionando resultados visíveis no que diz respeito aos movimentos, melhora da cicatrização e regeneração do colágeno. O gás carbônico vai agir quimicamente na modulação das interleucinas pró-inflamatórias, reduzindo a sua ação e aumentando a ação das interleucinas antiinflamatórias, o que leva um processo cicatricial normal. Simultaneamente, em razão do efeito mecânico do gás, haverá ruptura do colágeno cicatricial, que se encontra duro, 
denso e aderente às camadas mais profundas, e sua posterior substituição.

Quanto à prevenção de queimaduras, o presente estudo após a leitura dos artigos analisados mostra que apesar da eficácia das ações preventivas ser conhecida, os pacientes não recebem informações sobre queimaduras nem mesmo nas unidades de saúde. Então devese destacar a importância de educação populacional continuada, como meio de coibir acidentes evitáveis facilitados por: manipulação inadequada de elementos inflamáveis, ingestão concomitante de substâncias neurotrópicas, negligência quanto ao uso de equipamentos de proteção individual e imprudência nas atividades domiciliares e cuidados dispensados às crianças (GIMENES et al, 2009).

\section{CONCLUSÃO}

Pode-se concluir com esta revisão sistemática da literatura que a lesão inalatória é considerada a principal complicação do paciente queimado e a oxigenoterapia é muito utilizada para este tratamento. Houve alterações respiratórias significativas em pacientes com curativos oclusivos em tórax e as pressões do cuff alteram significativamente e acabam por expor o paciente a riscos causados por pressões inadequadas. Pessoas vítimas de queimaduras apresentam diminuição da qualidade de vida, sendo importante a reabilitação. $\mathrm{O}$ laser terapêutico tem a capacidade de induzir a cicatrização rápida, sendo um recurso valioso para o paciente queimado. A carboxiterapia não teve efeitos significativos. Deve-se destacar a importância da educação populacional continuada para a prevenção das queimaduras.

A fisioterapia atua em todas as consequências da queimadura, não apenas na parte motora e respiratória, mas também na parte psicológica dos indivíduos, tendo excelentes resultados para a recuperação do paciente queimado. Sendo essencial a intervenção precoce, ainda no ambiente hospitalar, para que se obtenham os resultados esperados de recuperação e cicatrização e minimização das sequelas tanto físicas como psicológicas desse indivíduo.

\section{REFERÊNCIAS}

ALBUQUERQUE, MLL; SILVA， GPF; DINIZ， DMSM; FIGUEIREDO, AMF; CÂMARA, TMF; BASTOS, VPD. Análise dos pacientes queimados com sequelas motoras em um hospital de referência na cidade de Fortaleza CE. Rev Bras Queimaduras. 2010;9(3):89-94

ANDRADE, AG; LIMA, CF;

ALBUQUERQUE, AKB. Efeitos do laser terapêutico no processo de cicatrização das queimaduras: uma revisão bibliográfica. $\mathbf{R e v}$

Bras Queimaduras. 2010;9(1):21-30. 
BORGES F. S. Modalidades terapêuticas nas

disfunções estéticas. 2a ed. Revisada e

ampliada. São Paulo. Phorte; 2010.

COSTA MCS; ROSSI, LA; LOPES, LM;

CIOFFI, CL. Significados de qualidade de vida: análise interpretativa baseada na experiência de pessoas em reabilitação de queimaduras. Rev

Latino-am Enfermagem 2008 março-abril; $16(2)$

DAIBEM, CGL; CONTI, TGT; SILVA, MMA; ROCHA, C. Análise das variações da pressão do cuff em paciente grande Queimado. Rev

Bras Queimaduras. 2011;10(1):21-6.

FERREIRA, TCR; CAREPA, SS; SPINELLI, JL; BASTOS, JO; COSTA, LR. Avaliação da mecânica respiratória em pacientes queimados com curativo oclusivo. Rev Bras

Queimaduras. 2011; 10(2):50-6.

GIMENES G. A; ALFERES F. C. B. A; DORSA P. P; BARROS A. C. P; GONELLA H. A. Estudo epidemiológico de pacientes internados no Centro de Tratamento de Queimados do Conjunto Hospitalar de Sorocaba. Ver Bras Queimaduras. 2009;8 (1):14-7.

GUIMARÃES, IBA; MARTINS, ABT; GUIMARÃES, SB. Qualidade de vida de pacientes com queimaduras internados em um hospital de referência no nordeste brasileiro.

Rev Bras Queimaduras. 2013;12(2):103-7.

JULIANO, SRR; JULIANO, MCR;

CIVIDANES, JP; HOULY, JGS; GEBARA, OCE; CIVIDANES, GVL;CATÃO, EC.

Medidas dos Níveis de Pressão do Balonete em Unidade de Terapia Intensiva: Considerações sobre os Benefícios do Treinamento. Revista Brasileira de Terapia Intensiva Vol. 19 No 3, Julho-Setembro, 2007.

JÚNIOR, GFP; VIEIRA, ACP; ALVES, GMG. Avaliação da qualidade de vida de indivíduos queimados pós alta hospitalar. Rev Bras Queimaduras. 2010;9(4):140-5.

LAMBERTI, DB; ARANTES, DP; OURIQUE, AAB; PRADO, ALC. Recursos

fisioterapêuticos em paciente Queimado: relato de caso de um sobrevivente Do incêndio na boate kiss. Revista UNINGÁ Review. Vol.18,n.2,pp.38-41 (Abr - Jun 2014).

LYRA, MC; JÚNIOR, HL; NETO, SP; ORGAES, FAFS; GONELLA, HÁ. Tratamento de queimaduras de carboxiterapia em modelo experimental. Rev Bras Queimaduras. 2012;11(1):2-5. MEDEIROS, VNLO. A fisioterapia respiratória diminui o tempo de 
permanência e ventilação mecânica de

pacientes internados na unidade de

tratamento intensivo? (Trabalho de conclusão

de mestrado) Rio De Janeiro 2010.

INSTITUTO BRASILEIRO DE TERAPIA

INTENSIVA.

SANTANA, CML; BRITO CF; COSTA,

ACSM. Importância da fisioterapia na reabilitação do paciente Queimado. Rev Bras

Queimaduras. 2012;11(4):240-5.

SILVA KP; CAPARRÓZ, MR;TORQUATO,

JÁ. Prevalência de complicações respiratórias

em pacientes com queimaduras internados num hospital público estadual de São Paulo. Rev

Bras Queimaduras. 2010; 9(4):130-5.

SOUZA, R. JARDIM, C; SALGE, JM;

CARVALHO, CRR. Lesão por inalação de

fumaça. J Bras Pneumol 2004; 30(5) 557-65

SOUZA T. R; SANTOS R. T; OLIVATTO R.

M. Treinamento muscular respiratório em lesão inalatória: relato de caso. Rev Bras

Queimaduras. 2009;8 (3):110-4.
SPINELLI, J; REZEGUE, L; FIORIN, R; BRAGANÇA, KR. Lesão inalatória grave: tratamento precoce e reversão do quadro. Relato de caso e revisão de literatura. Rev Bras Queimaduras. 2010;9(1):31-4.

TAKEJIMA, ML; NETTO, RFB; TOEBE, BL; ANDRETTA, MA; PRESTES, MA; TAKAKI, JL. Prevenção de queimaduras: avaliação do conhecimento sobre prevenção de queimaduras em usuários das unidades de saúde de Curitiba.

Rev Bras Queimaduras. 2011;10(3):85-8.

TORQUATO, JÁ; PARDAL, DMM;

LUCATO, JJJ; FU, C; GOMEZ, DS. O curativo compressivo usado em queimadura de tórax influencia na mecânica do sistema respiratório? Rev Bras Queimaduras. 2009;8(1):28-33.

VALE E. C. S. Primeiro atendimento em queimaduras: abordagem do dermatologista.

An Bras Dermatol. 2005;80 (1):9-1 